\title{
Relações da força muscular com indicadores de hipertrofia após 32 semanas de treinamento com pesos em mulheres na pós- menopausa
}

\author{
Muscle strength relation with hypertrophy indicators after 32 weeks of \\ resistance training in postmenopausal women
}

V. Bonganha, R.M.O. Botelho, M.S. Conceição, M.P.T. Chacon-Mikahil, V.A. Madruga

\begin{abstract}
RESUMO
O objectivo do presente estudo foi avaliar o comportamento da força muscular e a participação dos indicadores de hipertrofia, nos ganhos de força após 32 semanas de treinamento com pesos (TP), prescrito por zona alvo de repetições máximas, em mulheres na pós-menopausa. Participaram desta pesquisa 14 mulheres saudáveis e não ativas fisicamente. O TP teve frequência semanal de três vezes, em dias alternados. A composição corporal foi mensurada pela técnica das dobras cutâneas. Os indicadores de hipertrofia foram representados pela massa magra total e regional: área muscular do braço (AMB) e coxa (AMC). A força muscular foi avaliada pelo teste de uma repetição máxima nos exercícios leg press horizontal e rosca direta. Para análise estatística foi utilizado o teste de Friedman. Os resultados mostraram que a força muscular apresentou aumentos graduais e significantes durante a intervenção, que houve aumento da AMB e não houve diferença nos valores de AMC. A rosca direta mostrou forte associação com a AMB durante todos os momentos do estudo. Já o leg press pareceu estar mais efetivamente associado ao componente neural de ganhos de força, visto que a AMC não apresentou modificações significantes. Após 32 semanas de TP a força muscular aumentou significantemente, independentemente dos ganhos de massa magra.

Palavras-chave: força muscular, hipertrofia, pós-menopausa, exercício
\end{abstract}

ABSTRACT

The aim of the present study was to evaluate muscle strength and hypertrophy indicators on increases of strength after 32 weeks of resistance training (RT), prescribed by the target of maximum repetitions in postmenopausal women. Fourteen physically inactive healthy women participated in this study. Three times a week they participated on RT. Body composition was measured by the technique of skinfold thickness. The hypertrophy indicators were represented by total and regional lean mass: arm muscle area (AMA) and thigh muscle area (TMA). Muscle strength was assessed through the test of one maximum repetition in exercises of horizontal leg press and arm curl. Statistical analysis was performed using the Friedman test. Results showed increases in muscle strength, AMA but not in TMA. Arm curl was strongly correlated to AMA. Leg press seemed to be associated to neural components of increases in strength. Muscle strength significantly increased after 32 weeks of RT regardless of increases in lean mass.

Keywords: muscle strength, hypertrophy, postmenopausal, exercise

Submetido: 12.09 .2009 | Aceite: 29.03.2010

Valéria Bonganha e Miguel Soares Conceição. Programa de Pós-Graduação FEF/UNICAMP, Laboratório de Fisiologia do Exercício - FISEX.

Renata Maria de Oliveira Botelho. Laboratório de Fisiologia do Exercício - FISEX.

Mara Patrícia Traina Chacon-Mikahil e Vera Aparecida Madruga. Laboratório de Fisiologia do Exercício - FISEX, Faculdade de Educação Física - Universidade Estadual de Campinas.

Endereço para correspondência: Valéria Bonganha, Faculdade de Educação Física/Universidade Estadual de

Campinas - UNICAMP, Av. Érico Veríssimo, 701, Caixa Postal 6134, CEP: 13083-851 Campinas, Brasil.

E-mail: valeriabonganha@hotmail.com 
O hipoestrogenismo consiste na diminuição nos níveis de estrogênios durante a fase da menopausa, e é responsável por diversas mudanças que podem colocar em risco a saúde da mulher, dada a participação do hormônio feminino em diversos processos fisiológicos. $\mathrm{O}$ corpo da mulher sofre modificações como o aumento da gordura corporal e perda de massa magra (MM), levando a uma diminuição da força muscular incidindo, assim, num processo denominado sarcopenia (Trevisan \& Burini, 2007).

Quanto a isso, o treinamento com pesos (TP) tem sido recomendado para mulheres na pós-menopausa (Orsatti, Nahas, Maesta, Nahas-Neto, \& Burini, 2008; Trevisan \& Burini, 2007) por promover alterações hormonais e estruturais no músculo esquelético, proporcionando incremento na força e potência musculares e gerando também hipertrofia muscular, atuando assim na prevenção da sarcopenia (Kenny, Dawson, Kleppinger, Iannuzzi-Sucich, \& Judge, 2003).

Os ganhos de força muscular decorrentes do TP, inicialmente são atribuídos às adaptações neurais, como o aumento do recrutamento, sincronia e frequência de estimulação das unidades motoras, melhoria da coordenação dos grupos musculares antagonistas, com inibição de movimentos reflexos que prejudicam o movimento de ação primária (Sale, 1988). Posteriormente, a essa fase, a hipertrofia muscular passa a exercer progressivamente uma maior parcela de contribuição nos aumentos de força muscular (Phillips, 2000).

Contudo, se as alterações da composição corporal que ocorrem em mulheres na pósmenopausa podem influenciar a predominância dos componentes neurais e hipertróficos no aumento da força e MM, após intervenção com TP, é uma questão ainda não elucidada. Sendo assim, o objetivo do presente estudo foi verificar o comportamento da força muscular, e a participação dos indicadores de hipertrofia (MM total e regional), nos ganhos de força após 32 semanas de TP, em mulheres na pósmenopausa.

\section{MÉTODO}

\section{Amostra}

Participaram desta pesquisa, 14 voluntárias, clinicamente saudáveis e na pós-menopausa (ausência mínima de 12 meses de menstruações), com média de $55.92 \pm 4.92$ anos de idade e estatura média de $1.48 \pm .05$ metros. Como critérios de inclusão, as voluntárias não deveriam ser ativas fisicamente, não ter participado regularmente de nenhum programa de TP ao longo dos últimos seis meses precedentes ao início do experimento e não fazer uso de qualquer tipo de terapia de reposição hormonal.

Os critérios de exclusão foram patologias como: cardiopatias graves, obesidade mórbida, Diabetes Mellitus, problemas ósteomusculares severos e uso de qualquer medicação que pudesse interferir nas respostas fisiológicas dos testes.

Para tal, as voluntárias foram submetidas a exames clínicos realizados por médico especialista do Hospital das Clínicas da UNICAMP.

Todas as voluntárias foram aconselhadas a não mudar o padrão dos hábitos alimentares no decorrer da pesquisa, entretanto nenhum aconselhamento nutricional foi realizado.

Após serem conscientizadas sobre a proposta do estudo e procedimentos aos quais seriam submetidas, as voluntárias assinaram o consentimento livre e esclarecido.

Esse estudo foi aprovado pelo Comitê de Ética em Pesquisa da Universidade Estadual de Campinas, de acordo com as normas da Resolução 196/96 do Conselho Nacional de Saúde sobre pesquisa envolvendo seres humanos sob o protocolo $n^{\circ} 248 / 2004$, com adendo em 2007.

\section{Procedimentos}

A massa corporal total (MCT) foi mensurada numa balança de plataforma tipo Filizolla, (Brasil) e a medida da estatura foi obtida em 
um estadiômetro de madeira. A composição corporal foi obtida pela técnica de mensuração das espessuras das dobras cutâneas (DC) utilizando um adipômetro (LANGE Inglaterra). As dobras obtidas foram: subescapular, suprailíaca, abdominal, tricipital, bicipital, coxa, panturrilha e axilar média, segundo procedimentos descritos por Heyward e Stolarczyk (2000). A partir dos dados foram calculados a densidade corporal (Jackson, Pollock, \& Ward, 1980) e convertido o percentual (\%) de gordura por meio da fórmula de Siri (1961), permitindo a obtenção dos valores de massa magra e massa gorda.

A área muscular do braço (AMB) e a área muscular da coxa (AMC) foram calculadas de acordo com a equação de Frisancho (1984): AMB $\left(\mathrm{cm}^{2}\right)=[\mathrm{CBD}-(3.14 \times \mathrm{DCT})] / 4 \times \pi$, onde $\mathrm{CBD}=$ circunferência do braço direito em $\mathrm{cm}$, e DCT = dobra cutânea tricipital em $\mathrm{cm}$; AMC $\left(\mathrm{cm}^{2}\right)=\mathrm{CCX}-(\pi \times$ DCCX $)$. Onde $\mathrm{CCX}=$ circunferência da coxa direita em $\mathrm{cm}$, e DCCX $=$ DC da coxa direita em $\mathrm{cm}$.

Os indicadores de força muscular foram determinados por meio do teste de uma repetição máxima (1-RM) em dois exercícios, envolvendo membros inferiores e membros superiores, leg press horizontal e rosca direta, respectivamente. A padronização adotada foi conforme Clarke (1973).

Antes de executar cada exercício foi realizada uma série de aquecimento (10 repetições), com aproximadamente $50 \%$ da carga estimada para a primeira tentativa no teste de 1-RM. Após dois minutos do aquecimento, o teste era iniciado. As voluntárias foram orientadas a executar duas repetições. Caso fossem completadas as duas na primeira tentativa, ou mesmo se não fosse completada uma única repetição, uma segunda tentativa era executada após um intervalo de recuperação de três a cinco minutos. A carga da segunda tentativa era aumentada (caso fosse realizado duas repetições) ou diminuída (caso não fosse completada uma única repetição) àquela empregada na tentativa anterior.
Tal procedimento foi repetido novamente em uma terceira tentativa e caso ainda não fosse determinada a carga referente a uma única repetição máxima, uma nova sessão de teste era realizada após 48 horas. Caso a repetição máxima fosse encontrada na primeira ou na segunda tentativa, era dada oportunidade adicional para a voluntária, na expectativa de valores maiores ainda serem alcançados. O número máximo de tentativas foi fixado em três, para que a fadiga muscular gerada não comprometesse os resultados finais do teste. Portanto, a carga registrada como 1RM foi aquela na qual a voluntária conseguiu realizar uma única repetição (Clarke, 1973).

Embora testes de uma repetição máxima (1RM) sejam frequentemente utilizados para a avaliação da força muscular, acredita-se que os resultados obtidos possam ser afetados pela falta de familiarização prévia, até mesmo em sujeitos com experiência em exercícios com pesos, portanto foi realizado duas sessões de familiarização como sugerido no estudo divulgado por Dias et al. (2005a).

A intensidade do TP é geralmente baseada no teste de uma repetição máxima (1-RM) dos exercícios ou por zona alvo de repetição máxima (Fleck \& Kraemer, 2004), onde o número de repetições é fixado e não se baseia em valores percentuais de 1-RM. O modelo de prescrição de treinamento por zona alvo de RM não requer reavaliações periódicas para que se tenha um ajuste gradual de cargas (sobrecarga), além de gerar o mesmo número de repetições para todos os exercícios propostos no treinamento (Silva, Gurjão, Ferreira, Gobbi, \& Gobbi, 2006).

Os testes de 1-RM foram utilizados como meio de avaliação da força muscular, mas não como forma de prescrição da intensidade dos exercícios. O teste de 1-RM foi adotado devido aos estudos encontrados na literatura com a mesma população em questão, a fim de comparar os resultados aqui encontrados (Bonganha, Santos, Rocha, Chacon-Mikahil, Madruga, 2008; Orsatti et al., 2008; Trevisan \& Burini, 2007). 
O treinamento teve duração de 32 semanas, divididas em três fases: Fase 1 (F1), Fase 2 (F2) e Fase 3 (F3). As fases diferem-se quanto à intensidade, forma de ordenação e prescrição dos exercícios, como detalhadas a seguir.

$\mathrm{Na} F 1$, os exercícios foram realizados alternando membros superiores e tronco com membros inferiores, composto por três séries de 10 repetições máximas com intervalo de 1' entre as séries. Na F2, a ordem dos exercícios foi membros superiores e tronco e membros inferiores ou vice-versa, compostos por três séries de oito repetições máximas e intervalo entre as séries de 1'30". Na F3, a ordem dos exercícios foi a mesma da F2, composta por quatro séries de oito repetições máximas para exercícios de grandes grupos musculares e três séries de oito repetições máximas para pequenos grupos musculares e intervalo entre as séries de 1'. Essa prescrição seguiu as recomendações dos estudos apresentados pelo ACSM (2002).

Os exercícios propostos na F1 e F2 foram: mesa extensora; mesa flexora; supino horizontal com barra livre; puxada frente na polia; rosca direta com barra livre; extensão de tríceps com barra $\mathrm{W}$ na polia, leg press horizontal; abdominais, elevação lateral e panturrilha. Na F3, foi acrescido aos exercícios das etapas anteriores o leg press $45^{\circ}$, remada unilateral, supino com halteres, rosca alternada e tríceps testa com barra W. Para os exercícios abdominais e panturrilha foram prescritos três séries de 15 repetições durante todo o período experimental.

A intensidade da carga foi determinada por meio de zona alvo de RM e não em valores percentuais de 1-RM. As voluntárias foram sempre estimuladas a utilizarem uma carga que possibilitasse a realização do número de repetições estabelecidas (Bird, Tarpenning, \& Marino, 2005).

Foi utilizado reajuste semanal de carga, no qual foram realizadas duas séries de 8 ou 10 $\mathrm{RM}$, de acordo com a respectiva fase, e na terceira e última série as voluntárias realizavam o maior número de repetições possível, desde que a técnica de execução e amplitude do movimento fossem mantidos. Para cada repetição atingida além das RM propostas, era aumentado um quilo de carga para membro inferior e meio quilo para membro superior. Esse reajuste foi executado sempre no último dia de treino da semana, e tornou possível que o número de repetições fosse sempre mantido, entretanto com uma progressão gradativa da carga utilizada (Rodrigues \& Rocha, 1985).

Inicialmente foi empregado um protocolo de familiarização ao protocolo de treinamento no qual foram realizadas duas séries de 10 repetições em todos os exercícios propostos. Esta fase antecedeu os protocolos de avaliação de 1-RM, objetivando a não subestimação dos resultados obtidos (Dias et al., 2005a).

O TP foi realizado em três sessões semanais, em dias alternados e com duração de aproximadamente 60 minutos por sessão. As voluntárias foram supervisionadas por professores de Educação Física durante todo o período experimental, de modo que cada professor acompanhasse no máximo três voluntárias ao mesmo tempo.

O estudo teve uma duração de 37 semanas incluindo as fases de avaliações e treinamento, sendo que as avaliações foram distribuídas em pré, durante e após o TP, onde este teve duração de 32 semanas.

O TP foi dividido em quatro etapas todas com oito semanas de duração: E1 (semanas 2 a 9), E2 (semanas 11-18), da E3 (semanas 2027) e da E4 (semana 29 a 36).

As medidas e avaliações foram realizadas em cinco momentos: $\mathrm{MO}=$ semana um, antes do início do TP; $\mathrm{M} 1=$ semana 10 , após o término da E1; M2 = semana 19, após o término da E2; M3 = semana 28, após o término da E3; M4 = semana 37, após $\mathrm{O}$ término da E4. Nesses quatro momentos, as voluntárias não realizaram nenhum tipo de treinamento.

A tabela 1 apresenta a distribuição das etapas e momentos dos estudos. 
Tabela 1

Esquema que representa a distribuição das etapas e momentos do estudo

\begin{tabular}{cccccccccc}
\hline Momentos & M0 & & M1 & M2 & M3 & M4 \\
\hline Etapas & & E1 & & E2 & E3 & & E4 \\
\hline Semanas & 1 & 2 a 9 & 10 & 11 a 18 & 19 & 20 a 27 & 28 & 29 a 36 & 37 \\
\hline
\end{tabular}

\section{Análise estatística}

Para análise estatística dos dados foi utilizado o pacote estatístico "Bioestat", versão 5.0. O nível de significância adotado para todas as análises foi de $p<.05$. Para verificar a normalidade da amostra foi utilizado o teste de Shapiro-Wilks. Como não foi confirmada a normalidade dos dados foram utilizados testes não paramétricos. $O$ teste de Friedman foi utilizado para comparação dos resultados entre os momentos (M0, M1, M2, M3 e M4). A correlação de Spearman foi empregada para estabelecer relações entre as variáveis analisadas no estudo.

\section{RESULTADOS}

Os valores de MCT nos momentos do estudo estão apresentados na tabela 2. Não foram encontradas diferenças significantes entre os momentos.

Tabela 2

Valores médios e desvio padrão da massa corporal total em todos os momentos do estudo

\begin{tabular}{cccccc}
\hline Variáveis & M0 & M1 & M2 & M3 & M4 \\
\hline MCT $(\mathrm{kg})$ & $62.1 \pm 7.6$ & $62.2 \pm 7.9$ & $62.0 \pm 7.7$ & $62.0 \pm 6.8$ & $61.9 \pm 7.0$ \\
\hline
\end{tabular}

Os valores médios e desvio-padrão dos indicadores de força (teste de 1-RM no leg press) e hipertrofia muscular (AMC), encontrados nos diferentes momentos do estudo estão apresentados na tabela 3 .
A força muscular dos membros inferiores aumentou de maneira continua e significante $(p<.05)$ em todos os momentos, ao passo que a AMC não apresentou modificações estatisticamente significantes em nenhum momento do estudo.

Tabela 3

Valores médios e desvio-padrão do leg press (1-RM) e AMC, nos momentos do estudo

\begin{tabular}{cccccc}
\hline Variáveis & M0 & M1 & M2 & M3 & M4 \\
\hline Leg press $(\mathrm{kg})$ & $124.3 \pm 11.9$ & $140.4 \pm 14.6$ & $167.4 \pm 32.8$ & $187.3 \pm 36.3$ & $202.1 \pm 40.7$ \\
AMC $\left(\mathrm{cm}^{2}\right)$ & $45.6 \pm 3.2$ & $45.5 \pm 2.9$ & $46.0 \pm 3.0$ & $45.2 \pm 2.9$ & $45.3 \pm 2.9$ \\
\hline
\end{tabular}

A figura 1 mostra os valores do percentual (\%) de modificação entre os valores da força (1-RM no leg press) e hipertrofia muscular regional (AMC) entre as etapas do estudo.

Os resultados apresentados permitem verificar que a força muscular dos membros inferiores aumentou entre E1, E2, E3 e E4. O comportamento da AMC não foi semelhante ao da força, onde apresentou diminuição em E1 e
E3 e aumento em E2 e E4, embora essas diferenças não sejam estatisticamente significantes.

Os valores médios e desvio-padrão dos indicadores de força (teste de 1-RM na rosca direta) e hipertrofia muscular regional (AMB) encontrados nos diferentes momentos do estudo estão apresentados na tabela 4. 


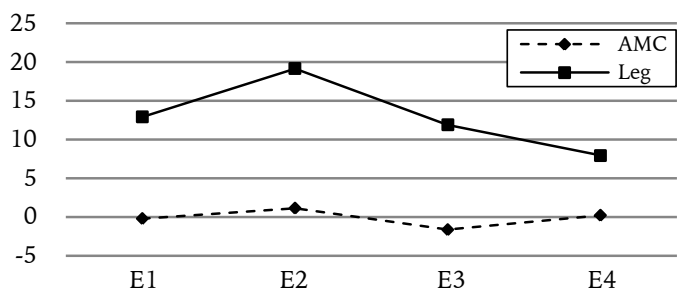

Figura 1. Valores do percentual de modificação entre as etapas do estudo para o leg press e AMC
A força muscular aumentou de maneira contínua e significante $(p<.05)$ em todas as etapas, ao mesmo tempo em que a $\mathrm{AMB}$ apresentou aumentos nos momentos M1, M2 e $\mathrm{M} 3$, em relação ao momento inicial (M0), exceto no M4.

Tabela 4

Valores médios e desvio-padrão da rosca direta (1-RM) e AMB entre os momentos do estudo

\begin{tabular}{cccccc}
\hline Variáveis & M0 & M1 & M2 & M3 & M4 \\
\hline Rosca $(\mathrm{kg})$ & $22.7 \pm 3.0$ & $25.5 \pm 2.6$ & $27.2 \pm 2.6$ & $28.9 \pm 2.9$ & $30.3 \pm 2.7$ \\
AMB $\left(\mathrm{cm}^{2}\right)$ & $28.4 \pm 1.7$ & $28.9 \pm 1.9$ & $29.1 \pm 1.6$ & $29.0 \pm 2.0$ & $28.9 \pm 2.0$ \\
\hline
\end{tabular}

A figura 2 mostra os valores do percentual (\%) de modificação entre os valores da força (1-RM na rosca direta) e hipertrofia muscular regional (AMB) entre as etapas do estudo.

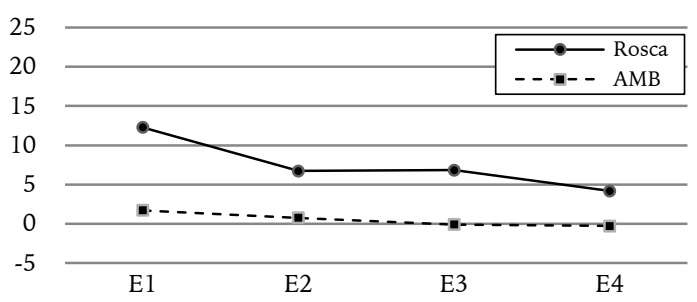

Figura 2. Valores do percentual de modificação entre as etapas do estudo para a rosca direta e AMB
A força muscular apresentou aumentos nas E1, E2 e E3 e E4. A AMB apresentou aumentos em E1 e E2.Os valores médios e desvio-padrão dos indicadores de força $(\Sigma$ - força) e hipertrofia muscular total (MM) encontrados nos diferentes momentos do estudo estão apresentados na tabela 5 .

A força muscular aumentou de maneira continua e significante $(p<.05)$ em todas as etapas, ao passo que a $\mathrm{MM}$ apresentou aumentos significantes entre M2 e M4, em relação a M0, e M2 e M3 em relação a M4.

Tabela 5

Valores médios e desvio-padrão do $\sum$-força (1-RM) e da MM entre os momentos do estudo

\begin{tabular}{cccccc}
\hline Variáveis & M0 & M1 & M2 & M3 & M4 \\
\hline$\sum$ - força $(\mathrm{kg})$ & $181.0 \pm 15.5$ & $205.0 \pm 16.3$ & $238.1 \pm 35.6$ & $263.9 \pm 41.2$ & $282.7 \pm 46.3$ \\
MM (kg) & $41.8 \pm 4.1$ & $42.2 \pm 4.1$ & $42.8 \pm 4.0$ & $42.2 \pm 4.0$ & $44.5 \pm 4.2$ \\
\hline
\end{tabular}

A figura 3 mostra os valores do percentual (\%) de modificação entre os valores do $\Sigma$ força (1-RM) e hipertrofia muscular total (MM) entre as etapas do estudo.

A força muscular apresentou aumentos nas E1, E2, E3 e E4. A MM também apresentou aumentos entre E1, E2 e E4 e uma diminuição na E3.

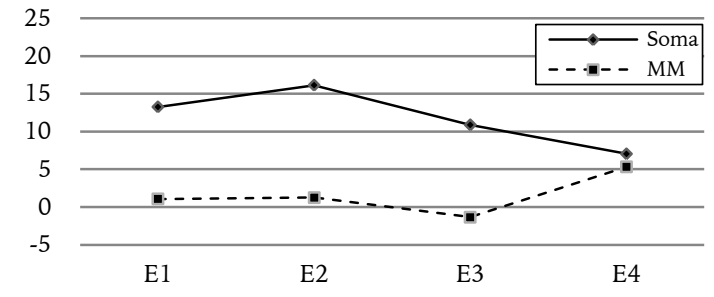

Figura 3. Valores do percentual de modificação entre as etapas do estudo para o $\sum$ de força e MM total 
A tabela 6 mostra os valores de coeficiente de correlação $(r)$ e índice de determinação $\left(r^{2}\right)$ e os respectivos valores de significância, entre os indicadores de força muscular (leg press, rosca direta e $\sum$ - força) e os indicadores de hipertrofia muscular total (MM) e regional (AMB, AMC) entre os momentos do estudo.

A correlação entre a AMB e a rosca direta apresentou valores estatisticamente significantes $(p<.05)$ em todos os momentos,
M0 $(r=.63)$, M1 $(r=.67)$, M2 $(r=.71)$, M3 $(r=.56)$ e M4 $(r=.64)$.

A AMC e o leg press não apresentaram valores de correlação significantes nos momentos analisados no estudo.

Para a correlação entre a MM e o $\sum$ - força foram encontrados valores estatisticamente significantes $(p<.05)$ nos momentos M3 $(r=$ $.58)$ e M4 $(r=.53)$.

Tabela 6

Coeficiente de correlação de Spearman e índice de determinação entre os indicadores de força e indicadores de hipertrofia nos diferentes momentos do programa

\begin{tabular}{ccccc}
\hline \multicolumn{2}{c}{ Correlações } & AMB $\times$ Rosca & AMC $\times$ Leg & MM $\times \sum$ - força \\
\hline \multirow{2}{*}{ M0 } & $r$ & $.63^{*}$ & .31 & .43 \\
& $r^{2}$ & 39.0 & 9.6 & 18.5 \\
M1 & $r$ & $.67^{*}$ & .38 & .16 \\
& $r^{2}$ & 44.0 & 14.4 & 2.1 \\
M2 & $r$ & $.71^{* *}$ & .20 & .49 \\
& $r^{2}$ & 50.0 & 4.0 & 24.0 \\
\multirow{2}{*}{ M3 } & $r$ & $.56^{*}$ & .42 & $.58^{*}$ \\
& $r^{2}$ & 31.0 & 17.6 & 32.4 \\
\multirow{2}{*}{ M4 } & $r$ & $.64^{*}$ & .35 & $.53^{*}$ \\
& $r^{2}$ & 40.0 & 12.2 & 27.9 \\
\hline
\end{tabular}

* Correlação significante, $p<.05 ;{ }^{* *}$ Correlação significante, $p<.01$

\section{DISCUSSÃO}

O objetivo desse estudo foi analisar o comportamento da força muscular, e a concomitante participação de indicadores de hipertrofia muscular (MM total e regional) nos ganhos de força, após 32 semanas de TP, estabelecendo possíveis associações entre eles.

Os resultados encontrados apontam aumentos graduais significantes e contínuos nos indicadores de força muscular (leg press, rosca direta e $\Sigma$ - força) em relação ao momento inicial, evidenciando a eficácia do TP proposto, em relação à prescrição por zona alvo e também pela progressão de carga utilizada.

Ocorre uma remodelação na musculatura para se adaptar as demandas impostas pelo exercício físico. Os estímulos extracelulares que chegam à membrana e interagem como receptores, ativando as vias de sinalização intracelulares, as quais resultam em alterações na transcrição gênica e síntese protéica.

A adaptação muscular às cargas impostas pelo exercício pode ativar as células satélites, que são células envolvidas no reparo e regeneração resultante de danos locais nas fibras musculares. Elas são capazes de ativar programas miogênicos, permitindo o reparo das fibras e hipertrofia muscular (Fernandes et al., 2008). Essas adaptações tornam possível o aumento da força muscular, independentemente da hipertrofia muscular.

Hakkinen et al. (1998) e Moritani e DeVries (1979) sugerem existir uma estabilização dos ganhos de força a partir do momento em que o componente neural do treinamento perde importância. Ao mesmo tempo que outros achados, que não encontraram platô nos ganhos de força após 12 semanas de TP, em 
homens idosos (Frontera, Meredith, O'Reilly, Knuttgen, \& Evans, 1988).

Não existe na literatura um consenso a respeito da participação dos componentes neurais e hipertróficos nos ganhos de força na população em processo de envelhecimento, pelo que os achados de vários estudos (Hakkinen et al, 1998; Moritani \& DeVries, 1979; Frontera et al., 1988) aumentam a disparidade das informações encontradas nas investigações.

Vários mecanismos estão envolvidos no processo de hipertrofia muscular frente ao exercício físico. O remodelamento do músculoesquelético é um processo dinâmico e responsivo a sinais extracelulares mediados pelo treinamento físico, atividade neural, hormônios, fatores de crescimento e citocinas. $\mathrm{O}$ aumento da MM é compreendido como balanço positivo entre a síntese e degradação protéica, realizado pela coordenação integrada da complexa rede de vias de sinalização intracelular. Entretanto, qual é o fator determinante para a hipertrofia muscular induzidas pelo TP na população em envelhecimento é uma questão ainda não elucidada.

A AMB e a rosca direta apresentaram correlações significantes entre si em todos os momentos analisados, mostrando que a MM da região do braço parece ter grande participação nos níveis de força alcançados nessa região.

Já a AMC e o leg press não foi encontrado comportamento semelhante após as 32 semanas de TP, visto que não foram encontradas correlações significantes entre eles em nenhum momento do estudo, sugerindo que os níveis de força alcançados no leg press não foram proporcionais à MM dessa região.

Esses resultados suportam a hipótese que a hipertrofia muscular tende a não ocorrer de forma uniforme nos diferentes grupos musculares (Abe, Kojima, Kearns, Yohena, \& Fukuda, 2003). As diferenças encontradas nos resultados dos indicadores de hipertrofia muscular podem estar relacionadas à dimensão das estruturas envolvidas na execução dos movimentos no leg press e rosca direta e no volume da massa muscular envolvida no exercício.

Os ganhos de força iniciais no leg press podem ser explicados pelo aspecto neuromotor, onde os ganhos de força não são prioritariamente dependentes da hipertrofia muscular (Frontera et al., 1988). O componente neural responsável pelos ganhos de força iniciais pode ser explicado pelo aumento da coordenação, tanto intra, como intermuscular durante a execução do movimento (Sale, 1988).

O aumento do recrutamento e sincronização de unidades motoras, a melhoria da coordenação dos grupos musculares antagonistas, o aumento da frequência de estimulação podem ser os fatores responsáveis pelo aumento significante da força muscular nos momentos iniciais do treinamento (McCarthy, Pozniak, \& Agre, 2002).

Exercícios que dependem de um menor número de articulações envolvidas, como rosca direta, pode ter uma contribuição maior dos componentes neurais, sendo que estes, aparentemente cessam mais precocemente (Dias et al., 2005b) exigindo uma maior contribuição dos componentes hipertróficos para ganhos adicionais de força muscular (Chilibeck, Calder, Sale, \& Webber, 1998).

Okano et al. (2008) encontraram resultados semelhantes em seu estudo de 24 semanas de TP realizado em homens jovens, onde nas primeiras oito semanas os componentes neurais foram os responsáveis pelo ganho de força e após esse período o componente hipertrófico passou a contribuir de maneira mais efetiva nos ganhos de força. Esses resultados reforçam a equidade das adaptações entre homens e mulheres de diferentes idades, perante um programa de TP. A MM total mostrou estar associada aos ganhos de força somente após 24 semanas de treinamento.

Ressalta-se que as intensidades da carga de treino nos dois exercícios (leg press e rosca direta), neste estudo, foram semelhantes devido à prescrição por zona alvo de RM, visto 
que a prescrição em relação a percentuais de 1RM pode gerar um número de repetições diferentes para diversos exercícios, provocando intensidades diferenciadas entre os grupamentos musculares envolvidos (Shimano et al., 2006).

O TP tem sido apontado uma alternativa para a população em processo de envelhecendo como tentativa de amenizar as perdas decorrentes desse processo (Kenny et al., 2003; Orsatti et al., 2008; Silva et al., 2006; Trancoso \& Farinatti, 2002). O TP induz a uma série de respostas fisiológicas agudas para ganhos de força e MM; alterações hormonais que promovem um ajuste no processo anabólico e alterações importantes nas adaptações crônicas induzidas pelo treinamento (Raastad, Bjùro, \& HalleÂn, 2000).

A pesquisa realizada por Barbosa et al., (2002) sobre TP em mulheres idosas mostrou que, aumentando progressivamente a intensidade do $\mathrm{TP}$, verifica-se um aumento significativo na força muscular.

Há na literatura, escassez de estudos com longos períodos de treinamento para mulheres na pós-menopausa para que fossem comparados os resultados do presente estudo. Os estudos encontrados envolvem períodos inferiores como 12 semanas (Silva et al., 2006; Trancoso \& Farinatti, 2002) e 16 semanas de TP (Kenny et al., 2003; Orsatti et al., 2008; Trevisan \& Burini, 2007).

Há grande importância em ressaltar as limitações e lacunas dessa pesquisa. A primeira dificuldade a ser levantada é a escassez de estudos científicos semelhantes para a comparação dos resultados. Em seguida destacamos a precisão das medidas dos indicadores de hipertrofia que são duplamente indiretas, embora bastante utilizadas (Barbosa, Santarém, Filho, \& Marucci, 2002; Okano et al., 2008). Deve-se atentar as limitações dessas medidas ao comparar a métodos mais sofisticados e consistentes nos resultados, como pelas técnicas de DEXA e biópsia muscular.
Após 32 semanas de TP, o aumento da força muscular de membros superiores foi proporcional às mudanças nos indicadores e hipertrofia do braço, ao passo que a força de membros inferiores aumentou significantemente, independentemente dos ganhos de MM. As diferentes respostas encontradas para membros superiores e inferiores podem estar relacionadas ao tamanho da massa muscular envolvida em cada região analisada.

O TP pode ser efetivo para a melhora da força muscular, independentemente da ocorrência concomitante de hipertrofia muscular em mulheres na pós-menopausa, podendo, assim, diminuir a ocorrência de quedas e fraturas em consequência da diminuição da força muscular presente nessa fase da vida.

\section{REFERÊNCIAS}

Abe, T., Kojima, K., Kearns, C. F., Yohena, H., \& Fukuda, J. (2003). Whole body muscle hypertrophy from resistance training: Distribution and total mass. British Journal of Sports Medicine 37(1), 543-545.

American College of Sports Medicine (2002). Position stand: Progression models in resistance training for healthy adults. Medicine and Science in Sports and Exercise 34 (2), 364-380.

Barbosa, A. R., Santarém, J. M., Filho, W. J., \& Marucci, M. F. N. (2002). Effects of resistance training on the sit-and-reach test in elderly women. The Journal of Strength and Conditioning Research 16(1), 14-18.

Bird, S. P., Tarpenning, K. M., \& Marino, F. E. (2005). Designing resistance training programmes to enhance muscular fitness: A review of the acute programme variables. Medicine and Science in Sports and Exercise, 35(10), 841-851.

Bonganha, V., Santos, C. F., Rocha, J., ChaconMikahil, M. P. T., \& Madruga, V. (2008). Força muscular e composição corporal de mulheres na pós-menopausa: Efeitos do treinamento concorrente. Revista Brasileira de Atividade Física e Saúde, 13(2), 102-109.

Chilibeck, P. D., Calder, A. W., Sale, D. G., \& Webber, C. E. (1998). A comparison of strength and muscle mass increases during resistance training in young women. European 
Journal Applied Physiology Occupational Physiology, 77(2), 170-175.

Clarke, D. H. (1973). Adaptations in strength and muscular endurance resulting from exercise. In J. H. Wilmore (Ed.), Exercise and sports sciences reviews (pp. 73-102) New York: Academic Press.

Dias, R. M. R., Cyrino, E. S., Salvador, E. P., Caldeira, L. F. S., Nakamura, F. Y., Papst, R. R., et al. (2005a). Influência do processo de familiarização para a avaliação dos níveis de força muscular em testes de 1-RM. Revista Brasileira de Medicina do Esporte, 11 (1), 34-38.

Dias, R. M. R., Cyrino, E. S., Salvador, E. P., Nakamura, F. Y., Pina, F. L. C., \& Oliveira, A. R. (2005b). Impacto de oito semanas de treinamento com pesos sobre a força muscular de homens e mulheres. Revista Brasileira de Medicina do Esporte, 11 (4), 224-228.

Fernandes, T., Soci, U. P. R., Alves, C. R., Carmo, E. C., Barros, J. G., \& Oliveira, E. M. (2008). Determinantes moleculares da hipertrofia do músculo esquelético mediados pelo treinamento físico: Estudo de vias de sinalização. Revista Mackenzie de Educação Física e Esporte, 7(1), 169-188.

Fleck, S. J. \& Kraemer, W. J. (2004) Designing resistance training programs ( $3^{\mathrm{a}}$ ed.). Champaign, IL: Human Kinetics.

Frisancho, A. R. (1984). New standards of weight and body composition by frame size height for assessment of nutritional status of adults and the elderly. American Journal of Clinical Nutrition, 40, 808-819.

Frontera, W., Meredith, C. N., O'Reilly, K. P., Knuttgen, H. G., \& Evans, W. J. (1988). Strength conditioning in older men: Skeletal muscle hypertrophy and improve function. Journal of Applied Physiology, 64(3), 1038-1044.

Hakkinen, K., Kallinen, M., Izquierdo, M., Jokelainen, K., Lassila, H., Malkia, E., et al. (1998). Changes in agonist-antagonist EMG, muscle CSA, and force during strength training in middle-aged and older people. Journal of Applied Physiology, 84(4), 1341-1349.

Heyward, V. H., \& Stolarczyk, L. M. (2000). Avaliação da composição corporal aplicada. São Paulo: Manole.

Jackson, A. S., Pollock, M. L., \& Ward, A. (1980). Generalized equations for predicting body density of women. Medicine and Science in Sports and Exercise, 12(3), 175-182.
Kenny, A. N., Dawson, L., Kleppinger, A., IannuzziSucich, M., \& Judge, J. O. (2003). Prevalence of sarcopenia and predictors of skeletal muscle mass in no-obese women who are long-term users of estrogen-replacement therapy. Journal of Gerontology, 58(5), 436-440.

McCarthy, J. P., Pozniak, M. A., \& Agre, J. C. (2002). Neuromuscular adaptations to concurrent strength and endurance training. Medicine and Science in Sports and Exercise, 34(3), 511-519.

Moritani, T., \& DeVries, H. (1979). Neural factors versus hypertrophy in the time course of muscle strength gain. American Journal of Physical Medicine, 58(3), 115-129.

Okano, A. H., Cyrino, E. S., Nakamura, F. Y., Guariglia, D. A., Nascimento, M. A., Avelar, A., et al. (2008). Comportamento da força muscular e da área muscular do braço durante 24 semanas de treinamento com pesos. Revista Brasileira de Cineantropometria e Desempenho Humano, 10(4), 379-385.

Orsatti, F. L., Nahas, E. A. P., Maesta, N., NahasNeto, J., \& Burini, R. C. (2008). Plasma hormones, muscle mass and strength in resistance-trained postmenopausal women. Maturitas, 59(4), 394-404.

Phillips, S. M. (2000). Short-term training: When do repeated bouts of resistance exercise become training? Canadian Journal of Applied Physiology, 25(3), 185-193.

Raastad, T., Bjùro, T., \& HalleÂn, J. (2000). Hormonal responses to high and moderate intensity strength exercise. European Journal of Applied Physiology, 82, 121-128.

Rodrigues, C. C., \& Rocha, P. E. C. P. (1985). Musculação: Teoria e prática. Rio de Janeiro: Sprint.

Sale, D. G. (1988). Neural adaptation to resistance training. Medicine and Science in Sports and Exercise, 20(5S), S135-145.

Shimano, T., Kraemer, W. J., Spiering, B. A., Volek, J. S., Hatfield, D. L., Silvestre, R., Vingren, M. S., et al. (2006). Relationship between the number of repetitions and selected percentages of one repetition maximum in free weight exercises in trained and untrained men. The Journal of Strength and Conditioning Research, 20, 819-823.

Silva, C. M., Gurjão, A. L. D., Ferreira, L., Gobbi, L. T. B., \& Gobbi, S. (2006). Efeito do treinamento com pesos, prescrito por zona de 
repetições máximas, na força muscular e composição corporal em idosas. Revista Brasileira de Cineantropometria e Desempenho Humano, 8(4), 29-45.

Siri, W. E. (1961). Body composition from fluid spaces and density: Analysis of methods. In J. Brozek \& A. Henschel (Eds.), Techniques for measuring body composition (pp. 223-244). Washington, DC: National Academic of Science.
Trancoso, E. S. F., \& Farinatti, P. T. V. (2002). Efeitos de 12 semanas de treinamento com pesos sobre a força muscular de mulheres com mais de 60 anos de idade. Revista Paulista de Educação Física, 16(2), 220-229.

Trevisan, M. C., \& Burini, R. C. (2007). Metabolismo de repouso de mulheres pósmenopausadas submetidas a programa de treinamento com pesos (hipertrofia). Revista Brasileira de Medicina do Esporte, 13 (2), 133-137. 